\title{
Defect-Induced Electronic Structure Changes in Cesium Lead Halide Nanocrystals
}

Arashdeep Singh Thind ${ }^{1}$, Jordan A. Hachtel ${ }^{2}$, Guangfu Luo ${ }^{3,4}$, Maria V. Morrell ${ }^{5}$, Yangchuan Xing ${ }^{5}$, Juan Carlos Idrobo ${ }^{2}$ and Rohan Mishra ${ }^{3,1}$

1. Institute of Materials Science \& Engineering, Washington University in St. Louis, St. Louis MO, USA.

2. Center for Nanophase Materials Sciences, Oak Ridge National Laboratory, Oak Ridge, TN, USA.

3. Department of Mechanical Engineering \& Materials Science, Washington University in St. Louis, St. Louis, MO, USA.

4. Department of Materials Science and Engineering, Southern University of Science and Technology, Guangdong Sheng, China.

5. Department of Chemical Engineering, University of Missouri, Columbia, MO, USA.

Lead-halide perovskites have emerged as promising candidates for applications in solar cells, lasing and solid-state lighting [1]. Most of the work on the defect physics of these materials has focused on point defects. It is now accepted that point defects with low formation enthalpy lead to shallow defect levels within the band gap [2]. However, the solution-based process used to synthesize these perovskites also results in a large concentration of planar defects that can play a critical role on charge transport and recombination. In our previous work, we used a novel post-synthesis process to trigger fusion in assynthesized $\mathrm{CsPbBr}_{3}$ nanocrystals [3]. Subsequently, using a combination of aberration-corrected scanning transmission electron microscope (STEM) imaging and density-functional-theory (DFT) calculations, we resolved the exact atomic structure of prevalent planar defects, including previously

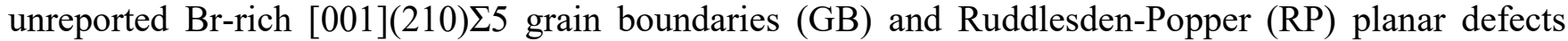
(shown in Figures 1a and 1b respectively) [4]. Moreover, using DFT calculations, we revealed that neither of these defects induce mid-gap states, but their Br-deficient counterparts do, where Br vacancies lead to mid-gap states because of $\mathrm{Pb}$ dangling bonds. Based on the electronic band diagrams of $\Sigma 5$ GBs and RPplanar defects, as shown in Figures $1 \mathrm{c}$ and 1d respectively, we find that the $\Sigma 5$ GBs repel electrons and attract holes while the RP-planar defects repel both electrons and holes. While combining DFT calculations with STEM imaging is a powerful technique to interpret electronic-structure changes around defects imaged with atomic-scale resolution using STEM, direct experimental observations of changes in the electronic structure around planar defects in lead-halide perovskites is still missing.

Recent advances in monochromated electron energy loss spectroscopy (EELS) have enabled access to the optical transition levels in the low-loss regime with an energy resolution of $<10 \mathrm{meV}$ while maintaining sub-Ångstrom spatial resolution [5]. We aim to leverage these developments in low-loss EELS to directly investigate changes in the electronic structure of $\mathrm{CsPbBr}_{3}$ nanocrystals at atomic-scale around planar defects. For instance, as shown in Figure 1d, DFT calculations predict that the RP-planar defect in $\mathrm{CsPbBr}_{3}$ acts as a semiconductor-insulator-semiconductor junction with a theoretical band gap widening of $\sim 327$ $\mathrm{meV}$ at the RP-planar defect boundary. The RP-planar defect, is therefore predicted to act as an effective barrier to the transport of both electrons and holes, impose strong quantum confinement effects, and results in high photoluminescence yield [3]. However, direct experimental confirmation is lacking along with the magnitude of the band gap change, which is often underestimated using DFT. We also aim to study the effect of Br-concentration on the electronic structure of these planar defects by carrying out in-situ STEM experiments, where we plan to systematically create $\mathrm{Br}$ vacancies by long time exposure of the $\mathrm{Br}$ atomic columns to the electron beam. The experimental understanding of the role of $\mathrm{Br}$ vacancies at the planar defects can enable optimization of the synthesis process towards targeted applications. Results discussing 
changes in electronic structure due to local composition changes in mixed-halide perovskites will also be presented. Overall, local electronic structure mapping with atomic-scale resolution in semiconductors, including near defects and interfaces, can enable higher performance with better control over the structure and properties [6].

References:

[1] CC Stoumpos and MG Kanatzidis, Adv. Mater. 28 (2016), p. 5778.

[2] W-J Yin, T Shi and Y Yan, Appl. Phys. Lett. 104 (2014), p. 063903.

[3] MV Morrell et al., ACS Applied Nano Materials 1 (2018), p. 6091.

[4] AS Thind et al., Adv. Mater. 31 (2019), p. e1805047.

[5] OL Krivanek et al., Ultramicroscopy (2018). doi: 10.1016/j.ultramic.2018.12.006.

[6] AST and RM acknowledge support from National Science Foundation (NSF DMR-1806147). A portion of the microscopy research was performed as part of a user proposal at Oak Ridge National Laboratory's (ORNL) Center for Nanophase Materials Sciences (CNMS), which is a U.S. Department of Energy (DOE), Office of Science User Facility. This research was conducted, in part, using instrumentation within ORNL's Materials Characterization Core provided by UT-Battelle, LLC under Contract No. DE-AC05-00OR22725 with the U.S. DOE. This work used the computational resources of XSEDE, which is supported by NSF grants ACI-1053575 and ACI-1548562.

a

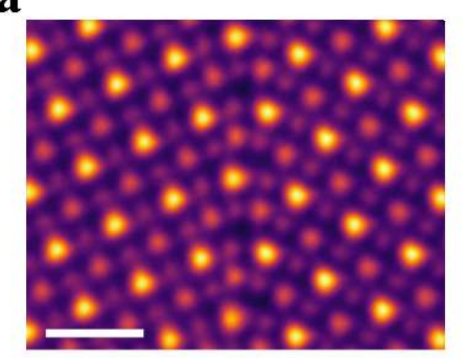

c

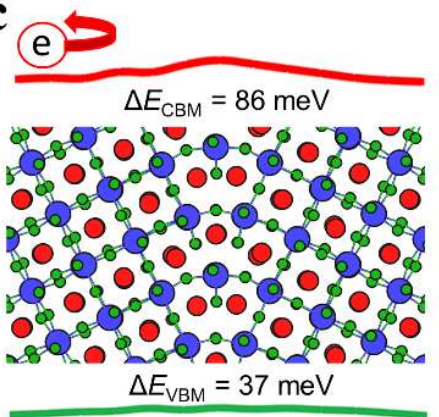

$-h \rightarrow$ b

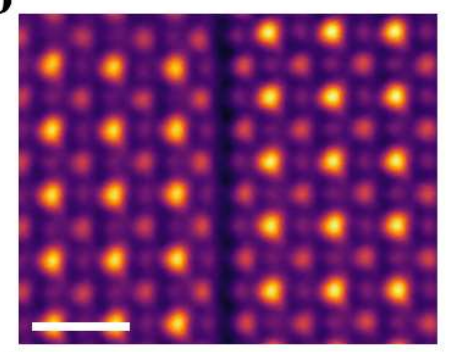

d
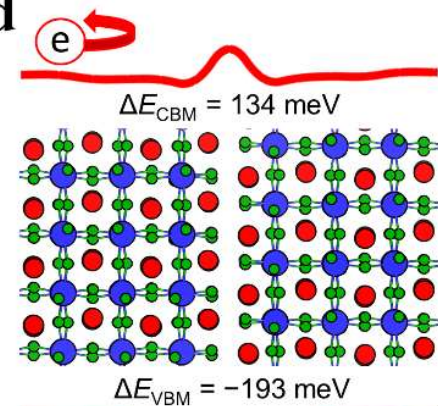

(h)

Figure 1. STEM Z-contrast images showing (a) $\Sigma 5 \mathrm{~GB}$ and (b) RP-planar defect in $\mathrm{CsPbBr}_{3}$ nanocrystals, where the brightest atomic columns corerespond to $\mathrm{Pb}$ followed by $\mathrm{Cs}$ and $\mathrm{Br}$. Scale bars correpsond to 1 $\mathrm{nm}$. DFT electronic band diagrams of (c) $\Sigma 5 \mathrm{~GB}$ and (d) RP-planar defect along with their respective DFT optimized structures. The blue, red and green atomic columns correspond to $\mathrm{Pb}, \mathrm{Cs}$ and $\mathrm{Br}$ respectively. 